\title{
Exponential synchronization and stabilization of delayed feedback hyperchaotic financial system
}

\author{
Ruofeng Rao ${ }^{1,2}$ and Quanxin Zhu ${ }^{3 *}$ (D)
}

"Correspondence: zqx22@126.com

${ }^{3}$ School of Mathematics and Statistics, Hunan Normal University, Changsha, China

Full list of author information is available at the end of the article

\begin{abstract}
In a real financial market, the delayed market feedback and the delayed effect of government macrocontrol are inevitable, and both bring mathematical difficulties in studying stabilization and synchronization of the hyperchaotic financial system. However, employing the Lyapunov function method, differential mean value theorem, and suitable bounded hypotheses and pulse control technology result in globally asymptotic stabilization and synchronization criteria. It is the first paper driving the stabilization and synchronization criteria under the assumptions of double delays. Finally, numerical examples illustrate the effectiveness of the proposed methods.
\end{abstract}

Keywords: Delayed feedback financial system; Hyperchaotic system; Differential mean value theorem; Synchronization; Impulse

\section{Introduction}

The analysis and control of financial chaotic system has become a hot topic (see, e.g., [110]), which is composed of production, labor, and currency subblocks. The generalization types of financial systems involve various economic problems and have been widely studied in the literature. For example, Harshavarthini, Sakthivel, Ma, and Muslim [11] designed a resilient fault-tolerant guaranteed cost controller with delay to tackle the fluctuations in investment policy scheme with minimum guaranteed cost bound and also to achieve finite-time boundedness. Different from that of [11], we investigate a chaotic financial system with average profit rate, which is in fact a hyperchaotic financial system introduced in [12]. Based on the improved financial chaos system model, the authors of [12] considered the global economic crisis caused by American subprime mortgage crisis in 2007 as the background of the market model, adding a state variable (average profit rate) to the chaotic financial system and proposed a new kind of hyperchaotic financial systems.

On the other hand, many results on delay have been reported in the literature (e.g., see [13-22]). In fact, both the market feedback delay and the delayed effect of the government macroeconomic control are inevitable, but seldom papers involved the above-mentioned multiple delays, which inspired us to write this paper. For the first time, the stabilization

(c) The Author(s) 2021. This article is licensed under a Creative Commons Attribution 4.0 International License, which permits use sharing, adaptation, distribution and reproduction in any medium or format, as long as you give appropriate credit to the original author(s) and the source, provide a link to the Creative Commons licence, and indicate if changes were made. The images or other third party material in this article are included in the article's Creative Commons licence, unless indicated otherwise in a credit line to the material. If material is not included in the article's Creative Commons licence and your intended use is not permitted by statutory regulation or exceeds the permitted use, you will need to obtain permission directly from the copyright holder. To view a copy of this licence, visit http://creativecommons.org/licenses/by/4.0/. 
and synchronization are simultaneously considered for the hyperchaotic financial systems with double delays. The newly obtained results will provide some theoretical guidance for the actual financial market.

The rest of this paper is arranged as follows. In Sect. 2, we present some system descriptions and preparations. Inspired by some methods of [20-22], we derive the main results on stabilization and synchronization in Sect. 3. Besides, in Sect. 4, we present two numerical examples to illustrate the effectiveness of the new criteria. In the final section, we derive some conclusions to further elaborate the main purpose and significance of this paper.

\section{System descriptions and preparations}

The following hyperchaotic financial system was introduced in [12]:

$$
\left\{\begin{array}{l}
\dot{x}=z+(y-a) x+u \\
\dot{y}=1-b y-x^{2} \\
\dot{z}=-x-c z \\
\dot{u}=-d x y-k u
\end{array}\right.
$$

which has three equilibrium points:

$$
P_{0}\left(0, \frac{1}{b}, 0,0\right), \quad P_{1,2}\left( \pm \theta, \frac{k+a c k}{c(k-d)}, \mp \frac{\theta}{c}, \frac{d \theta(1+a c)}{c d-c k}\right) \quad \text { with } \theta=\sqrt{\frac{k b+a b c k}{c(d-k)}+1}
$$

Since the delayed feedback is a common phenomenon in real markets, we get the following delayed feedback model for system (2.1):

$$
\left\{\begin{array}{l}
\dot{x}=z+(y-a) x+u+k_{1}\left(x-x\left(t-\tau_{1}(t)\right)\right) \\
\dot{y}=1-b y-x^{2}+k_{2}\left(y-y\left(t-\tau_{2}(t)\right)\right) \\
\dot{z}=-x-c z+k_{3}\left(z-z\left(t-\tau_{3}(t)\right)\right) \\
\dot{u}=-d x y-k u+k_{4}\left(u-u\left(t-\tau_{4}(t)\right)\right)
\end{array}\right.
$$

where $a$ represents savings, $b$ represents the unit investment cost, and $c$ represents the elasticity of commodity demand, $d$ and $k$ are nonlinear and linear feedback coefficients, respectively, $x$ represents the interest rate, $y$ represents the investment demand, $z$ represents the price index, $u$ represents the average profit margin, $\tau_{1}(t), \tau_{2}(t), \tau_{3}(t)$, and $\tau_{4}(t)$ represent the time delays of the interest rate, investment demand, and price index at time $t$, respectively, and $k_{i}$ are the corresponding feedback gain coefficients.

Let $X=\left(x_{1}, x_{2}, x_{3}, x_{4}\right)^{T}$, and let

$$
\left\{\begin{array}{l}
x_{1}=x-\theta, \\
x_{2}=y-\frac{k+a c k}{c(k-d)}, \\
x_{3}=z+\frac{\theta}{c} \\
x_{4}=u-\frac{d \theta(1+a c)}{c d-c k} .
\end{array}\right.
$$


then the equilibrium point $P_{1}\left(\theta, \frac{k+a c k}{c(k-d)},-\frac{\theta}{c}, \frac{d \theta(1+a c)}{c d-c k}\right)$ with positive interest rate $\theta>0$ of system (2.2) corresponds to the zero solution of the following system:

$$
\left\{\begin{array}{l}
\dot{x_{1}}=x_{3}-\frac{\theta}{c}+\left(x_{2}+\frac{k+a c k}{c(k-d)}-a\right)\left(x_{1}+\theta\right)+x_{4}+\frac{d \theta(1+a c)}{c d-c k}+k_{1}\left(x-x\left(t-\tau_{1}(t)\right)\right), \\
\dot{x_{2}}=1-b\left(x_{2}+\frac{k(a c k}{c(k-d)}\right)-\left(x_{1}+\theta\right)^{2}+k_{2}\left(y-y\left(t-\tau_{2}(t)\right)\right), \\
\dot{x_{3}}=-\left(x_{1}+\theta\right)-c\left(x_{3}-\frac{\theta}{c}\right)+k_{3}\left(z-z\left(t-\tau_{3}(t)\right)\right), \\
\dot{x_{4}}=-d\left(x_{1}+\theta\right)\left(x_{2}+\frac{k+a c k}{c(k-d)}\right)-k\left(x_{4}+\frac{d \theta(1+a c)}{c d-c k}\right)+k_{4}\left(u-u\left(t-\tau_{4}(t)\right)\right),
\end{array}\right.
$$

or

$$
\left\{\begin{array}{l}
\dot{x_{1}}=\left(\frac{k+a c k}{c(k-d)}-a\right) x_{1}+\theta x_{2}+x_{3}+x_{4}+x_{1} x_{2}+k_{1}\left(x-x\left(t-\tau_{1}(t)\right)\right), \\
\dot{x_{2}}=-2 \theta x_{1}-b x_{2}-x_{1}^{2}+k_{2}\left(y-y\left(t-\tau_{2}(t)\right)\right), \\
\dot{x_{3}}=-x_{1}-c x_{3}+k_{3}\left(z-z\left(t-\tau_{3}(t)\right)\right), \\
\dot{x_{4}}=-\frac{d(k+a c k)}{c(k-d)} x_{1}-d \theta x_{2}-k x_{4}-d x_{1} x_{2}+k_{4}\left(u-u\left(t-\tau_{4}(t)\right)\right), \\
X(s)=\phi(s), \quad s \in[-\tau, 0],
\end{array}\right.
$$

where $X=\left(x_{1}, x_{2}, x_{3}, x_{4}\right)^{T}$. Furthermore, system (2.4) can be rewritten in the vector-matrix form

$$
\left\{\begin{array}{l}
\dot{X}(t)=A X(t)+f(X(t))+K(X(t)-X(t-\tau(t))), \quad t \geq 0, \\
X(s)=\phi(s), \quad s \in[-\tau, 0]
\end{array}\right.
$$

where

$$
\begin{aligned}
A & =\left(\begin{array}{cccc}
\left(\frac{k+a c k}{c(k-d)}-a\right) & \theta & 1 & 1 \\
-2 \theta & -b & 0 & 0 \\
-1 & 0 & -c & 0 \\
-\frac{d(k+a c k)}{c(k-d)} & -d \theta & 0 & -k
\end{array}\right), \quad f(X)=\left(\begin{array}{c}
x_{1} x_{2} \\
-x_{1}^{2} \\
0 \\
-d x_{1} x_{2}
\end{array}\right), \\
K & =\left(\begin{array}{cccc}
k_{1} & 0 & 0 & 0 \\
0 & k_{2} & 0 & 0 \\
0 & 0 & k_{3} & 0 \\
0 & 0 & 0 & k_{4}
\end{array}\right) .
\end{aligned}
$$

Remark 1 Obviously, $f(X)$ is not Lipschitz continuous.

Under the delayed impulse control on system (2.5), we can get

$$
\left\{\begin{array}{l}
\dot{X}(t)=A X(t)+f(X(t))+K(X(t)-X(t-\tau(t))), \quad t \geq 0, t \neq t_{k}, \\
X\left(t_{k}^{+}\right)-X\left(t_{k}^{-}\right)=D_{k} X\left(t_{k}-\rho_{k}\right), \quad k=1,2, \ldots, \\
X(s)=\phi(s), \quad s \in[-\tau, 0],
\end{array}\right.
$$


or

$$
\left\{\begin{array}{l}
\dot{x}=z+(y-a) x+u+k_{1}\left(x-x\left(t-\tau_{1}(t)\right)\right), \quad t \geq 0, t \neq t_{k}, \\
\dot{y}=1-b y-x^{2}+k_{2}\left(y-y\left(t-\tau_{2}(t)\right)\right), \quad t \geq 0, t \neq t_{k}, \\
\dot{z}=-x-c z+k_{3}\left(z-z\left(t-\tau_{3}(t)\right)\right), \quad t \geq 0, t \neq t_{k}, \\
\dot{u}=-d x y-k u+k_{4}\left(u-u\left(t-\tau_{4}(t)\right)\right), \quad t \geq 0, t \neq t_{k}, \\
X\left(t_{k}^{+}\right)-X\left(t_{k}^{-}\right)=D_{k} X\left(t_{k}-\rho_{k}\right), \quad k=1,2, \ldots, \\
X(s)=\phi(s), \quad s \in[-\tau, 0],
\end{array}\right.
$$

where $X$ is defined in (2.3). So the zero solution of system of (2.7) corresponds to the equilibrium point $P_{1}\left(\theta, \frac{k+a c k}{c(k-d)},-\frac{\theta}{c}, \frac{d \theta(1+a c)}{c d-c k}\right)$ of the hyperchaotic financial system (2.8).

Construct the following response system for the drive system (2.7):

$$
\left\{\begin{array}{l}
\dot{Y}(t)=A Y(t)+f(Y(t))+K(Y(t)-Y(t-\tau(t))), \quad t \geq 0, t \neq t_{k} \\
Y\left(t_{k}^{+}\right)-Y\left(t_{k}^{-}\right)=D_{k} Y\left(t_{k}-\rho_{k}\right), \quad k=1,2, \ldots, \\
Y(s)=\psi(s), \quad s \in[-\tau, 0],
\end{array}\right.
$$

and the error system of pulse synchronization is given as follows:

$$
\left\{\begin{array}{l}
\dot{e}(t)=A e(t)+\breve{f}(e(t))+K(e(t)-e(t-\tau(t))), \quad t \geq 0, t \neq t_{k}, \\
e\left(t_{k}^{+}\right)-e\left(t_{k}^{-}\right)=D_{k} e\left(t_{k}-\rho_{k}\right), \quad k=1,2, \ldots \\
e(s)=\psi(s)-\phi(s), \quad s \in[-\tau, 0]
\end{array}\right.
$$

where $e=\left(e_{1}, e_{2}, e_{3}\right)^{T}=\left(Y_{1}-X_{1}, Y_{2}-X_{2}, Y_{3}-X_{3}\right)^{T}$ is the error of synchronization, and

$$
\breve{f}(e)=f(Y)-f(X)=\left(\begin{array}{c}
y_{1} y_{2}-x_{1} x_{2} \\
x_{1}^{2}-y_{1}^{2} \\
0 \\
d x_{1} x_{2}-d y_{1} y_{2}
\end{array}\right)
$$

Lemma 2.1 ([23, Theorem 4.2]) Under the assumptions of [23, Theorem 4.1], the following fuzzy system (2.12) is bounded in the $L^{\infty}$ sense:

$$
\left\{\begin{aligned}
\dot{X}(t)= & \sum_{r=1}^{n} \varrho_{r}(\hat{\omega}(t)) H_{\sigma r} X(t)+f(X(t))-D_{\sigma} X(t) \\
& +\varphi_{\sigma}(X(t), X(t-\tau(t))) d w(t), \quad t \geq 0, \\
X(s)= & \xi(s), \quad s \in[-\tau, 0] .
\end{aligned}\right.
$$

For convenience, we employ the following notation:

- For a symmetric matrix $A$, we denote by $\lambda_{\max } A$ the maximum eigenvalue of $A$;

- For a vector $v=\left(v_{1}, v_{2}, v_{3}\right)^{T} \in R^{3}$, we denote by $\|v\|=\sqrt{\sum_{i=1}^{3} v_{i}^{2}}$ the norm of $v$;

- For a matrix $A$, we denote by $\|A\|=\sqrt{\lambda_{\max }\left(A^{T} A\right)}$ the norm of $A$.

\section{Stabilization and synchronization by impulse control}

First, in this section, we may assume that $X\left(t_{k}^{-}\right)=X\left(t_{k}\right)$ for all $k \in \mathbb{Z}^{+}$. Second, from Lemma 2.1 we can similarly derive the boundedness of systems (2.7) and (2.8) under mild 
conditions. In fact, the particular case of the fuzzy system in Lemma 2.1 is a common system without any fuzzy factors, and the boundedness result of Lemma 2.1 includes this particular case

$$
\left\{\begin{array}{l}
\dot{x}=z+(y-a) x, \\
\dot{y}=1-b y-x^{2}, \\
\dot{z}=-x-c z .
\end{array}\right.
$$

So we can utilize the methods in the proof of [23, Theorem 4.2] to prove the boundedness of system (2.1), (2.2), (2.7), or (2.9). In this paper, we introduce the following boundedness assumptions:

(H1) The time delays $\tau_{i}(t) \in[0, \tau], i=1,2,3$, and there are two positive scalars $M_{1}, M_{2}$ such that

$$
0<M_{1} \leq\|X(s)\|^{2} \leq M_{2}, \quad s \in[-\tau,+\infty) .
$$

If the upper limit of delays $\tau$ is appropriately small, the following continuity hypothesis is natural due to the boundedness:

(H2) For any $\tau>0$, there exists the corresponding positive number $c_{\tau}>0$ such that

$$
\left|\|X(t)\|^{2}-\|X(t-\tau(t))\|^{2}\right|<c_{\tau} .
$$

In this section, we assume that the time delays $\tau(t) \in[-\tau, 0]$ and $\rho_{k} \in[-\rho, 0]$ for all $k \in \mathbb{Z}^{+}$. To obtain the stability of the system, a certain pulse frequency is required, so we assume a smaller pulse interval as follows:

$$
\sup _{k \in \mathbb{Z}^{+}}\left(t_{k}-t_{k-1}\right)<c_{0}
$$

where $c_{0}$ is a positive number.

Theorem 3.1 Suppose that conditions (H1), (H2), and (3.3) hold. If

$$
\begin{aligned}
& {\left[\left\|D_{k}+I\right\|+\rho_{k}\left\|D_{k}\right\| \cdot\left(\|A\|+\sqrt{M_{2}\left(1+d^{2}\right)}+2\|K\| \frac{\sqrt{M_{2}}}{\sqrt{M_{1}}}\right)\right] e^{\frac{c_{\lambda} c_{0}}{2}} \leq d_{0}<1,} \\
& \quad \forall k \in \mathbb{Z}^{+}
\end{aligned}
$$

where $d_{0}$ is a positive scalar,

$$
c_{\lambda}=\lambda_{\max }\left[A+A^{T}+2 K+r_{1} I+\frac{1}{r_{1}} M_{2}\left(1+d^{2}\right) I+r_{2} K^{2}+\frac{1}{r_{2}}\left(1+\frac{c_{\tau}}{M_{1}}\right) I\right],
$$

I represents the identity matrix, and $\rho_{k}<t_{k}-t_{k-1}$ for all $k \in \mathbb{Z}^{+}$, then the zero solution of system (2.7) is globally asymptotically stable, and the equilibrium solution $P_{1}$ with positive interest rate $\theta$ of system (2.8) is globally asymptotically stable. 
Proof Consider the Lyapunov function

$$
V(t)=\|X\|^{2} .
$$

It follows from $(\mathrm{H} 1)$ that

$$
\|f(X)\|^{2} \leq M_{2}\left(1+d^{2}\right)\|X\|^{2} .
$$

Next, (H1) and (H2) yield

$$
\begin{aligned}
D^{+} V(t, X)= & 2 X^{T}[A X(t)+f(X(t))+K(X(t)-X(t-\tau(t)))] \\
\leq & X^{T}\left[A+A^{T}+2 K+r_{1} I+\frac{1}{r_{1}} M_{2}\left(1+d^{2}\right) I\right] X \\
& +\left[r_{2}|X|^{T} K^{2}|X|+\frac{1}{r_{2}}|X(t-\tau(t))|^{T}|X(t-\tau(t))|\right] \\
\leq & \lambda_{\max }\left[A+A^{T}+2 K+r_{1} I+\frac{1}{r_{1}} M_{2}\left(1+d^{2}\right) I+r_{2} K^{2}+\frac{1}{r_{2}}\left(1+\frac{c_{\tau}}{M_{1}}\right) I\right] \\
& \times V(t, X), \quad t \in\left(t_{k-1}, t_{k}\right], k \in \mathbb{Z}^{+} .
\end{aligned}
$$

Then we get

$$
\|X(t)\|^{2} \leq\left\|X\left(t_{k-1}^{+}\right)\right\|^{2} e^{c_{\lambda}\left(t-t_{k-1}\right)}, \quad t \in\left(t_{k-1}, t_{k}\right] .
$$

According to the impulsive condition, we have

$$
X\left(t_{k}^{+}\right)=X\left(t_{k}^{-}\right)+D_{k} X\left(t_{k}-\rho_{k}\right)=X\left(t_{k}\right)+D_{k} X\left(t_{k}-\rho_{k}\right) .
$$

So we can conclude that there exist $\eta_{k i}(i=1,2,3)$ with $\eta_{k i} \in\left(t_{k}-\rho_{k}, t_{k}\right)$ such that

$$
\begin{aligned}
\left\|X\left(t_{k}^{+}\right)\right\|= & \left\|\left(D_{k}+I\right) X\left(t_{k}\right)-\rho_{k} D_{k} \dot{X}\left(\eta_{k}\right)\right\| \\
\leq & \left\|D_{k}+I\right\| \cdot\left\|X\left(t_{k}\right)\right\| \\
& +\rho_{k}\left\|D_{k}\right\| \cdot\left\|A X\left(\eta_{k}\right)+f\left(X\left(\eta_{k}\right)\right)+K\left(X\left(\eta_{k}\right)-X\left(\eta_{k}-\tau\left(\eta_{k}\right)\right)\right)\right\| \\
\leq & \left\|D_{k}+I\right\| \cdot\left\|X\left(t_{k}\right)\right\| \\
& +\rho_{k}\left\|D_{k}\right\|\left[\left(\|A\|+\sqrt{M_{2}\left(1+d^{2}\right)}\right)\left\|X\left(\eta_{k}\right)\right\|+\|K\| \cdot\left\|X\left(\eta_{k}\right)-X\left(\eta_{k}-\tau\left(\eta_{k}\right)\right)\right\|\right] \\
\leq & {\left[\left\|D_{k}+I\right\|+\rho_{k}\left\|D_{k}\right\| \cdot\left(\|A\|+\sqrt{M_{2}\left(1+d^{2}\right)}+\|K\| \frac{2 \sqrt{M_{2}}}{\sqrt{M_{1}}}\right)\right] } \\
& \times e^{\frac{c_{2} c_{0}}{2}}\left\|X\left(t_{k-1}^{+}\right)\right\|,
\end{aligned}
$$

which, together with (3.4), implies that $\left\{\left\|X\left(t_{k}^{+}\right)\right\|\right\}_{k=1}^{\infty}$ is a convergent sequence with zero limit, where $X\left(\eta_{k}\right)=\left(x_{1}\left(\eta_{k 1}\right), x_{2}\left(\eta_{k 2}\right), x_{3}\left(\eta_{k 3}\right)\right)^{T}$. For any $t \in\left(t_{k}, t_{k+1}\right]$, by (3.9) we get

$$
0 \leq\|X(t)\| \leq\left\|X\left(t_{k-1}^{+}\right)\right\| e^{\frac{c_{\lambda}}{2}\left(t-t_{k-1}\right)} \leq e^{\frac{c_{\lambda}}{2} c_{0}}\left\|X\left(t_{k-1}^{+}\right)\right\| \rightarrow 0, \quad k \rightarrow \infty,
$$

which completes the proof. 
Remark 2 The condition $\rho_{k}<t_{k}-t_{k-1}$ implies that every macrocontrol measure (pulse) of the government should be effective enough to see the pulse effect within each pulse interval. Besides, the condition $\sup _{k \in \mathbb{Z}^{+}}\left(t_{k}-t_{k-1}\right)<c_{0}$ guarantees a pulse (macrocontrol) of certain frequency if $c_{0}>0$ is appropriately small. No matter how complex and chaotic the financial system is, high-frequency active macrocontrol is conducive to the globally asymptotic stability of the economic system.

Remark 3 In comparison with the existing related literature $[5,6,8,9,12]$, in this paper, there are some innovations in financial models and research methods. For example, $\mathrm{Yu}$, Cai, and Li [12] employed the speed feedback control and linear feedback control to stabilize a hyperhaotic system. However, the delayed effects of impulse are not investigated, and the effects of macrocontrol in economy are often delayed in actual finance markets. Our Theorem 3.1 demonstrates how to stabilize the economic system near the equilibrium point with positive interest rate by setting an appropriate pulse interval and pulse intensity, which is one of the main innovations of this paper.

To derive the synchronization criterion, we may consider the following boundedness assumptions:

(H3) There are two positive scalars $N_{1}, N_{2}$ such that

$$
0<N_{1} \leq\|e(s)\|^{2} \leq N_{2}, \quad s \in[-\tau,+\infty) .
$$

(H4) For any $\tau>0$, there exists the corresponding positive number $d_{\tau}>0$ such that

$$
\left|\|e(t)\|^{2}-\|e(t-\tau(t))\|^{2}\right|<d_{\tau} .
$$

Theorem 3.2 Assume that $X, Y$ satisfy boundedness conditions $(\mathrm{H} 1)-(\mathrm{H} 2)$ and that the error variable e satisfies boundedness assumptions (H3)-(H4). If, in addition,

$$
\left[\left\|D_{k}+I\right\|+\rho_{k}\left\|D_{k}\right\| \cdot\left(\|A\|+\sqrt{4 M_{2}\left(2+d^{2}\right)}+\|K\| \frac{2 \sqrt{N_{2}}}{\sqrt{N_{1}}}\right)\right] e^{\frac{d_{\lambda} c_{0}}{2}} \leq d_{0}<1,
$$

then system (2.9) can be globally exponentially synchronized onto system (2.7), where

$$
d_{\lambda}=\lambda_{\max }\left[A+A^{T}+2 K+r_{1} I+\frac{4}{r_{1}} N_{2}\left(2+d^{2}\right) I+r_{2} K^{2}+\frac{1}{r_{2}}\left(1+\frac{d_{\tau}}{N_{1}}\right) I\right] .
$$

Proof Consider the Lyapunov function

$$
V(t)=\|e\|^{2} .
$$

Similarly, from the assumptions of Theorem 3.2 we can conclude that

$$
\begin{aligned}
\|\breve{f}(e)\|^{2} & \leq 2\left(1+d^{2}\right)\left[\|X\|^{2}+\|Y\|^{2}\right]\|e\|^{2}+2\left(\|X\|^{2}+\|Y\|^{2}\right)\|e\|^{2} \\
& \leq 4\left(1+d^{2}\right) M_{2}\|e\|^{2}+4 M_{2}\|e\|^{2}=4\left(2+d^{2}\right) M_{2}\|e\|^{2}
\end{aligned}
$$


and

$$
\begin{aligned}
D^{+} V(t, e)= & 2 e^{T}(t)[A e(t)+\breve{f}(e(t))+K(e(t)-e(t-\tau(t)))] \\
\leq & e^{T}\left[A+A^{T}+2 K+r_{1} I+\frac{4}{r_{1}} N_{2}\left(2+d^{2}\right) I+r_{2} K^{2}+\frac{1}{r_{2}}\left(1+\frac{d_{\tau}}{N_{1}}\right) I\right] e \\
\leq & \lambda_{\max }\left[A+A^{T}+2 K+r_{1} I+\frac{4}{r_{1}} N_{2}\left(2+d^{2}\right) I+r_{2} K^{2}+\frac{1}{r_{2}}\left(1+\frac{d_{\tau}}{N_{1}}\right) I\right] \\
& \times V(t, e), \quad t \in\left(t_{k-1}, t_{k}\right], k \in \mathbb{Z}^{+} .
\end{aligned}
$$

Then we get

$$
\begin{aligned}
& \|e(t)\|^{2} \leq\left\|e\left(t_{k-1}^{+}\right)\right\|^{2} e^{d_{\lambda}\left(t-t_{k-1}\right)} \\
& \quad \Rightarrow \quad\|X(t)\| \leq\left\|X\left(t_{k-1}^{+}\right)\right\| e^{\frac{d_{\lambda}}{2}\left(t-t_{k-1}\right)}, \quad t \in\left(t_{k-1}, t_{k}\right] .
\end{aligned}
$$

According to the impulsive condition, we have

$$
e\left(t_{k}^{+}\right)=e\left(t_{k}^{-}\right)+D_{k} e\left(t_{k}-\rho_{k}\right)=e\left(t_{k}\right)+D_{k} e\left(t_{k}-\rho_{k}\right) .
$$

So we deduce that there exist $\eta_{k i}(i=1,2,3)$ with $\eta_{k i} \in\left(t_{k}-\rho_{k}, t_{k}\right)$ such that

$$
\begin{aligned}
\left\|e\left(t_{k}^{+}\right)\right\| \leq & \left\|D_{k}+I\right\| \cdot\left\|e\left(t_{k}\right)\right\|+\rho_{k}\left\|D_{k}\right\| \cdot\left\|A e\left(\eta_{k}\right)+\breve{f}\left(e\left(\eta_{k}\right)\right)+K\left(e\left(\eta_{k}\right)-e\left(\eta_{k}-\tau\left(\eta_{k}\right)\right)\right)\right\| \\
\leq & \left\|D_{k}+I\right\| \cdot\left\|e\left(t_{k}\right)\right\| \\
& +\rho_{k}\left\|D_{k}\right\|\left[\left(\|A\|+\sqrt{4 M_{2}\left(2+d^{2}\right)}\right)\left\|e\left(\eta_{k}\right)\right\|+\|K\| \cdot\left\|e\left(\eta_{k}\right)-e\left(\eta_{k}-\tau\left(\eta_{k}\right)\right)\right\|\right] \\
\leq & \left\|D_{k}+I\right\| \cdot\left\|e\left(t_{k-1}^{+}\right)\right\| e^{\frac{d_{\lambda}}{2}\left(t-t_{k-1}\right)} \\
& +\rho_{k}\left\|D_{k}\right\|\left(\|A\|+\sqrt{4 M_{2}\left(2+d^{2}\right)}+\|K\| \frac{2 \sqrt{N_{2}}}{\sqrt{N_{1}}}\right)\left\|e\left(t_{k-1}^{+}\right)\right\| e^{\frac{d_{\lambda}}{2}\left(t-t_{k-1}\right)} \\
\leq & {\left[\left\|D_{k}+I\right\|+\rho_{k}\left\|D_{k}\right\| \cdot\left(\|A\|+\sqrt{4 M_{2}\left(2+d^{2}\right)}+\|K\| \frac{2 \sqrt{N_{2}}}{\sqrt{N_{1}}}\right)\right] } \\
& \times e^{\frac{d_{\lambda} c_{0}}{2}}\left\|e\left(t_{k-1}^{+}\right)\right\|,
\end{aligned}
$$

which, together with (3.10), implies that $\left\{\left\|e\left(t_{k}^{+}\right)\right\|\right\}_{k=1}^{\infty}$ is a convergent sequence with zero limit, where $e\left(\eta_{k}\right)=\left(x_{1}\left(\eta_{k 1}\right), x_{2}\left(\eta_{k 2}\right), x_{3}\left(\eta_{k 3}\right)\right)^{T}$. For any $t \in\left(t_{k}, t_{k+1}\right]$, by (3.15) we get

$$
0 \leq\|e(t)\| \leq\left\|e\left(t_{k-1}^{+}\right)\right\| e^{\frac{d_{\lambda}}{2}\left(t-t_{k-1}\right)} \leq e^{\frac{d_{\lambda}}{2} c_{0}}\left\|e\left(t_{k-1}^{+}\right)\right\| \rightarrow 0, \quad k \rightarrow \infty,
$$

which completes the proof.

Remark 4 In [24], on the basis of the adaptive control theory and Lyapunov stability function, a feedback linear control law and a parameter estimation law were designed. Then the proposed linear feedback controller scheme was evaluated by identical synchronization of the finance chaotic system with unknown system parameters. Although the synchronization of various chaotic finance systems was much studied in the literature, the delayed 
impulsive effect was not investigated with respect to its influence on synchronization effect (see, e.g., [7, 24, 25]). This problem is perfectly solved in our Theorem 3.2, which is another innovation of this paper.

Remark 5 To some extent, we use the method in [26] to deal with synchronization and stabilization on delayed feedback hyperchaotic financial systems of this paper. However, in this paper, system (2.7) involves two time delays $\tau(t)$ and $\rho_{k}$, whereas [26] only involved one time delay. Particularly, the function $f$ in system (2.7) does not originally satisfy the corresponding condition of [26], which brings about mathematical difficulty in utilizing the methods of [26]. We use [23, Theorem 4.2] as Lemma 2.1, so that we can propose the boundedness assumption (H1), and a series of mathematical analysis allows us to overcome this difficulty.

\section{Numerical examples}

Example 4.1 Consider system (2.7) or (2.8) with the following data:

$$
\begin{aligned}
& M_{1}=1, \quad M_{2}=8, \quad \tau=0.5, \quad c_{\tau}=0.3, \\
& \rho_{k} \equiv 0.01, \quad D_{k} \equiv-\frac{4}{5} I, \quad r_{1}=r_{2}=r_{3}=r_{4}=1, \\
& K=\left(\begin{array}{cccc}
0.05 & 0 & 0 & 0 \\
0 & 0.07 & 0 & 0 \\
0 & 0 & 0.03 & 0 \\
0 & 0 & 0 & 0.033
\end{array}\right) .
\end{aligned}
$$

We consider two different data for comparison.

Case 1. Let

$$
a=0.3, \quad b=0.2, \quad c=0.5, \quad d=0.11, \quad k=0.81, \quad c_{0}=0.15 .
$$

Then direct computation yields that $\theta=\sqrt{\frac{k b+a b c k}{c(d-k)}+1}=0.6839$ and

$$
A=\left(\begin{array}{cccc}
2.3614 & 0.6839 & 1.0000 & 1.0000 \\
-1.3678 & -0.2000 & 0 & 0 \\
-1.0000 & 0 & -0.5000 & 0 \\
-0.2928 & -0.0752 & 0 & -0.8100
\end{array}\right)
$$

Further computation gives $\|A\|=3.2952,\|K\|=0.07$, and $c_{\lambda}=18.0898$. Let $d_{0}=0.99$. Direct computation results in

$$
\begin{aligned}
& {\left[\left\|D_{k}+I\right\|+\rho_{k}\left\|D_{k}\right\| \cdot\left(\|A\|+\sqrt{M_{2}\left(1+d^{2}\right)}+2\|K\| \frac{\sqrt{M_{2}}}{\sqrt{M_{1}}}\right)\right] e^{\frac{c_{\lambda} c_{0}}{2}}} \\
& \quad=0.8021 \leq 0.99=d_{0}<1, \quad \forall k \in \mathbb{Z}^{+},
\end{aligned}
$$

which implies that condition (3.4) is satisfied, and thus the equilibrium solution $P_{1}$ with positive interest rate $\theta=68.39 \%$ of system (2.8) is globally asymptotically stable by Theorem 3.1. 
Case 2. Let

$$
a=0.9, \quad b=0.2, \quad c=0.5, \quad d=0.11, \quad k=0.81, \quad c_{0}=0.17 \text {. }
$$

Then direct computation yields that $\theta=\sqrt{\frac{k b+a b c k}{c(d-k)}+1}=0.5735$ and

$$
A=\left(\begin{array}{cccc}
2.4557 & 0.5735 & 1.0000 & 1.0000 \\
-1.1469 & -0.2000 & 0 & 0 \\
-1.0000 & 0 & -0.5000 & 0 \\
-0.3691 & -0.0631 & 0 & -0.8100
\end{array}\right)
$$

Further computation gives $\|A\|=3.2922,\|K\|=0.07$, and $c_{\lambda}=18.2325$. Let $d_{0}=0.99$. Direct computation results in

$$
\begin{aligned}
& {\left[\left\|D_{k}+I\right\|+\rho_{k}\left\|D_{k}\right\| \cdot\left(\|A\|+\sqrt{M_{2}\left(1+d^{2}\right)}+2\|K\| \frac{\sqrt{M_{2}}}{\sqrt{M_{1}}}\right)\right] e^{\frac{c_{\lambda} c_{0}}{2}}} \\
& \quad=0.9729 \leq 0.99=d_{0}<1, \quad \forall k \in \mathbb{Z}^{+},
\end{aligned}
$$

which implies that condition (3.4) is satisfied, and the equilibrium solution $P_{1}$ with positive interest rate $\theta=57.35 \%$ of system (2.8) is globally asymptotically stable by Theorem 3.1.

Remark 6 Table 1 shows that when the system becomes stable, the higher the interest rate, the smaller the pulse interval. This shows that to reach a balance of higher interest rates in the financial market, the government should speed up the pace of macrocontrol of the economy.

Example 4.2 Consider the following data for systems (2.7), (2.9) and (2.10):

$$
\begin{aligned}
& N_{1}=0.1, \quad N_{2}=0.5, \quad M_{1}=1, \quad M_{2}=8, \quad \tau=0.5, \\
& c_{\tau}=0.3, \quad \rho_{k} \equiv 0.001, \quad D_{k} \equiv-\frac{4}{5} I, \quad r_{1}=r_{2}=r_{3}=r_{4}=1, \\
& a=0.9, \quad b=0.2, \quad c=0.5, \quad d=0.11, \quad k=0.81, \quad c_{0}=0.01, \\
& K=\left(\begin{array}{cccc}
0.05 & 0 & 0 & 0 \\
0 & 0.07 & 0 & 0 \\
0 & 0 & 0.03 & 0 \\
0 & 0 & 0 & 0.033
\end{array}\right) .
\end{aligned}
$$

Table 1 Comparisons of positive interest rate and pulse interval

\begin{tabular}{lll}
\hline & Interest rate $\theta$ & Pulse interval $c_{0}$ \\
\hline Case 1 & $68.39 \%$ & 0.15 \\
Case 2 & $57.35 \%$ & 0.17 \\
\hline
\end{tabular}


Then

$$
A=\left(\begin{array}{cccc}
2.3614 & 0.6839 & 1.0000 & 1.0000 \\
-1.3678 & -0.2000 & 0 & 0 \\
-1.0000 & 0 & -0.5000 & 0 \\
-0.2928 & -0.0752 & 0 & -0.8100
\end{array}\right)
$$

Further computations give

$$
\begin{aligned}
& {\left[\left\|D_{k}+I\right\|+\rho_{k}\left\|D_{k}\right\| \cdot\left(\|A\|+\sqrt{4 M_{2}\left(2+d^{2}\right)}+\|K\| \frac{2 \sqrt{N_{2}}}{\sqrt{N_{1}}}\right)\right] e^{\frac{d_{\lambda} c_{0}}{2}}} \\
& \quad=0.9055 \leq 0.99=d_{0}<1,
\end{aligned}
$$

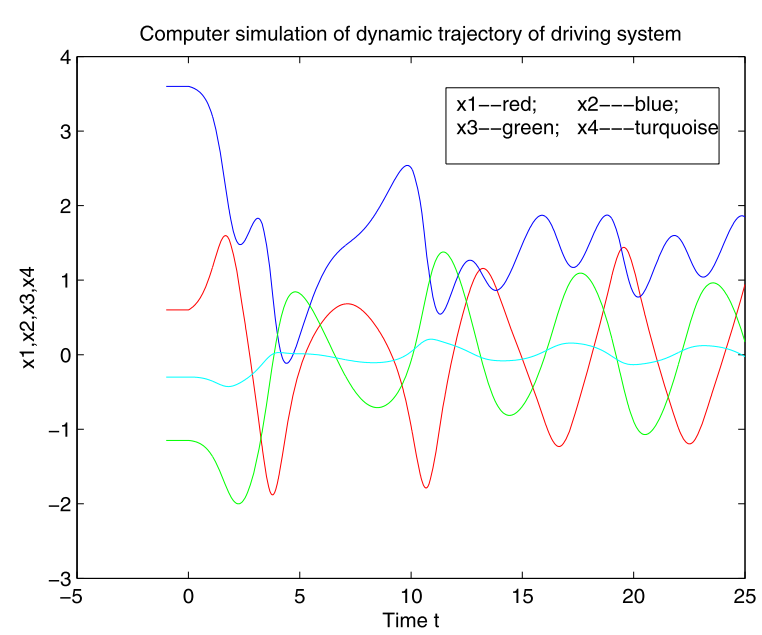

Figure 1 Dynamic trajectories of driving system

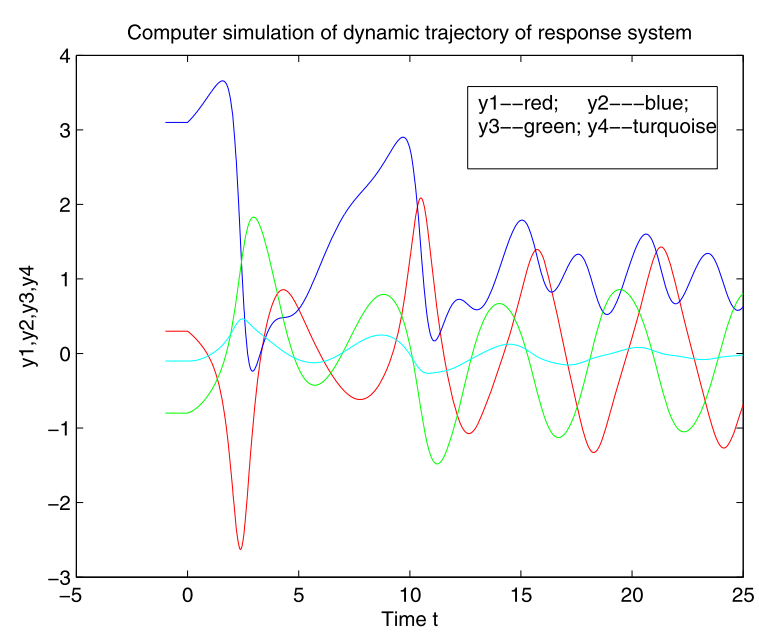

Figure 2 Dynamic trajectories of response system 
which implies that condition (3.10) holds. Hence Theorem 3.2 tells us that system (2.9) can be globally exponentially synchronized onto system (2.7) (see Figs. 1-2).

Remark 7 We can see from Figs. 1-2 that the dynamic trajectories of two systems are not completely the same due to different initial values, but the dynamic trajectories of response system are correspondingly approaching those of the driving system as time goes by. When the time variable $t$ becomes larger, the dynamic curves of the same color are almost identical. So Example 4.2 shows the feasibility of Theorem 3.2.

Remark 8 The dynamics are considered to be known for future direction. However, the authors in [27-29] proposed the potential estimation of uncertainties by the developed interval type-3 fuzzy systems and general type- 2 fuzzy systems. In our next paper, similar considerations may be placed in the chaotic financial system, which is an interesting thing.

\section{Conclusions and further considerations}

Interest rates are always positive at most of the countries in the world when the economies are in balance. So our Theorems 3.1 and 3.2 illustrate theoretical guidance significance for the actual financial market. In particular, Theorem 3.1 shows that positive and correct macroeconomic control measures with certain frequency are conducive to market balance and high positive interest rates. Finally, two numerical examples show the effectiveness and feasibility of stabilization and synchronization criteria.

In [30], a sliding-mode investment policy design is developed by solving the obtained linear matrix inequality (LMI)-based constrained algorithm on chaotic financial system, and some good ideas and techniques inspire us. How to employ the methods utilized in [30] to our delayed feedback hyperchaotic financial system? It is a desirable consideration in the future.

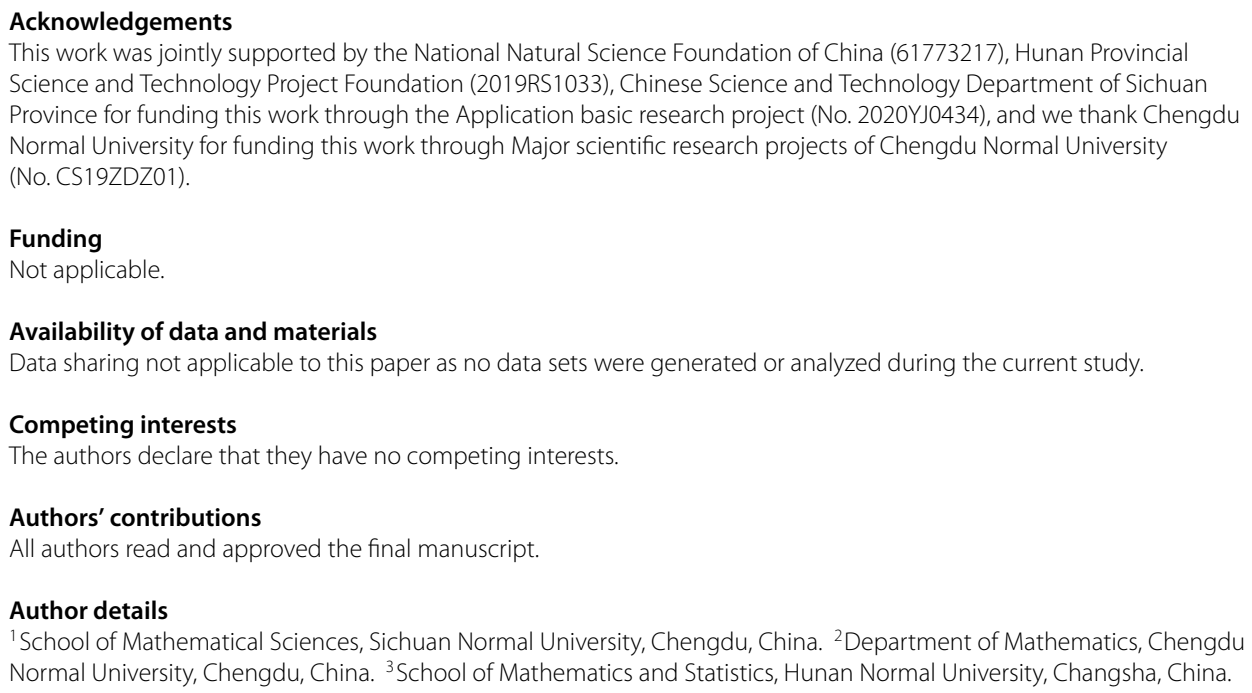




\section{References}

1. Cheng, S.: Complicated science and management. In: Article Collection of Beijing Xiangshan Conference, vol. 1. Science Press, Beijing (1998) (in Chinese)

2. Huang, D., Li, H.: Theory and Method of Nonlinear Economics. Sichuan University Press, Chengdu (1993) (in Chinese)

3. Ma, J., Chen, Y.: Study for the bifurcation topological structure and the global complicated character of a kind of nonlinear finance system (I). Appl. Math. Mech. 11, 1240-1251 (2001)

4. Ma, J., Chen, Y.: Study for the bifurcation topological structure and the global complicated character of a kind of nonlinear finance system (II). Appl. Math. Mech. 12, 1375-1382 (2001)

5. Chen, W.: Dynamics and control of a financial system with time-delayed feedbacks. Chaos Solitons Fractals 37(4), 1198-1207 (2008)

6. Zhao, M., Wang, J.: $H_{\infty}$ control of a chaotic finance system in the presence of external disturbance and input time-delay. Appl. Math. Comput. 233, 320-327 (2014)

7. Zhao, X., Li, Z., Li, S.: Synchronization of a chaotic finance system. Appl. Math. Comput. 217,6031-6039 (2011)

8. Rao, R:: Global stability of a Markovian jumping chaotic financial system with partially unknown transition rates under impulsive control involved in the positive interest rate. Mathematics 7(7), 579 (2019)

9. Rao, R., Zhong, S.: Impulsive control on delayed feedback chaotic financial system with Markovian jumping. Adv. Differ. Equ. 2020, 50 (2020)

10. Rao, R., Zhong, S.: Input-to-state stability and no-inputs stabilization of delayed feedback chaotic financial system involved in open and closed economy. Discrete Contin. Dyn. Syst., Ser. S 14(4), 1375-1393 (2021)

11. Harshavarthini, S., Sakthivel, R., Ma, Y., Muslim, M.: Finite-time resilient fault-tolerant investment policy scheme for chaotic nonlinear finance system. Chaos Solitons Fractals 132, 109567 (2020)

12. Yu, H., Cai, G., Li, Y.: Dynamic analysis and control of a new hyperchaotic finance system. Nonlinear Dyn. 67(3), 2171-2182 (2012)

13. Ali, M.S., Usha, M., Zhu, Q., Shanmugam, S.: Synchronization analysis for stochastic T-S fuzzy complex networks with Markovian jumping parameters and mixed time-varying delays via impulsive control. Math. Probl. Eng. 2020, 9739876 (2020)

14. Kong, F., Zhu, Q., Sakthivel, R., Mohammadzadeh, A.: Fixed-time synchronization analysis for discontinuous fuzzy inertial neural networks with parameter uncertainties. Neurocomputing 422, 295-313 (2021)

15. Cao, W., Zhu, Q.: Razumikhin-type theorem for $p$ th exponential stability of impulsive stochastic functional differential equations based on vector Lyapunov function. Nonlinear Anal. Hybrid Syst. 39, 100983 (2021)

16. Kong, F., Zhu, Q.: New fixed-time synchronization control of discontinuous inertial neural networks via indefinite Lyapunov-Krasovskii functional method. Int. J. Robust Nonlinear Control (2020). https://doi.org/10.1002/rnc.5297

17. Kong, F., Zhu, Q., Huang, T.: New fixed-time stability lemmas and applications to the discontinuous fuzzy inertial neural networks. IEEE Trans. Fuzzy Syst. (2020). https://doi.org/10.1109/TFUZZ.2020.3026030

18. Song, R., Wang, B., Zhu, Q.: Delay-dependent stability of nonlinear hybrid neutral stochastic differential equations with multiple delays. Int. J. Robust Nonlinear Control 31, 250-267 (2021)

19. Liu, X., Zhu, Q.: Stochastically globally exponential stability of stochastic impulsive differential systems with discrete and infinite distributed delays based on vector Lyapunov function. Complexity 2020, 7913050 (2020)

20. Rao, R., Zhu, Q., Shi, K.: Input-to-state stability for impulsive Gilpin-Ayala competition model with reaction diffusion and delayed feedback. IEEE Access 8, 222625-222634 (2020)

21. Saravanakumar, T., Muoi, N., Zhu, Q.: Finite-time sampled-data control of switched stochastic model with non-deterministic actuator faults and saturation nonlinearity. J. Franklin Inst. 357(18), 13637-13665 (2020)

22. Zhang, M., Zhu, Q.: Stability analysis for switched stochastic delayed systems under asynchronous switching: a relaxed switching signal. Int. J. Robust Nonlinear Control 30(18), 8278-8298 (2020)

23. Rao, R., Li, X.: Input-to-state stability in the meaning of switching for delayed feedback switched stochastic financial system. AIMS Math. 6, 1040-1064 (2021)

24. Tirandaz, H., Aminabadi, S., Tavakoli, H.: Chaos synchronization and parameter identification of a finance chaotic system with unknown parameters, a linear feedback controller. Alex. Eng. J. 57, 1519-1524 (2018)

25. Harshavarthini, S., Sakthivel, R., Kong, F.: Finite-time synchronization of chaotic coronary artery system with input time-varying delay. Chaos Solitons Fractals 134, 109683 (2020)

26. Zhang, Q., Lu, J., He, K.: Time-delayed impulsive control for a class of dynamical systems. Syst. Eng. Elect. 10 1686-1689 (2007) (in Chinese)

27. Mohammadzadeh, A., Sabzalian, M.H., Zhang, W.: An interval type-3 fuzzy system and a new online fractional-order learning algorithm: theory and practice. IEEE Trans. Fuzzy Syst. 28(9), 1940-1950 (2020)

28. Mohammadzadeh, A., Zhang, W.: Dynamic programming strategy based on a type-2 fuzzy wavelet neural network. Nonlinear Dyn. 95, 1661-1672 (2019)

29. Mohammadzadeh, A., Kayacan, E.: A novel fractional-order type-2 fuzzy control method for online frequency regulation in ac microgrid. Eng. Appl. Artif. Intell. 90, 103483 (2020)

30. Sakthivel, R., Kaviarasan, B., Selvaraj, P., Karimi, H.: ElD-based sliding mode investment policy design for fuzzy stochastic jump financial systems. Nonlinear Anal. Hybrid Syst. 31, 100-108 (2019) 